\title{
STRATEGIC PLANNING AND BUDGETING OF WILDLAND FIRE PREPAREDNESS PROGRAMS FOR RISK MANAGEMENT
}

\author{
D.B. RIDEOUT ${ }^{1}$, YU WEI ${ }^{1}$, A.G. KIRSCH ${ }^{2}$ \& N. KERNOHAN ${ }^{1}$ \\ ${ }^{1}$ WESTFIRE Research Center, Department of Forest and Rangeland Stewardship Colorado State University, \\ Fort Collins, Colorado. \\ ${ }^{2}$ US Department of Interior, National Park Service, National Interagency Fire Center, Boise, Idaho.
}

\begin{abstract}
In approaches that were previously used, preparedness planning used tactical modelling of individual fire events and resource deployment. Results from modelling individual fire events were typically gathered to construct a program analysis at the planning unit or at the landscape level. This research deviates from those tactical-based approaches by using strategic modelling to inform planning and budgeting decisions for a preparedness program. In preparedness planning and budgeting, wildland fire officials must consider the dual importance of reducing wildfire risk to highly valued resources (initial attack) and managing for the beneficial effects of wildland fire that can foster resilient fire-adapted ecosystems. This dual purpose requires the employment of a similar set of resources including crews, equipment and planning. Consequently, we address the allocation of a single 'preparedness' budget to the dual purpose of initial attack and beneficial wildland fire (BWF) using return on investment (ROI) as the performance metric.

Keywords: beneficial wild fires, initial attack, planning, preparedness, risk, wildland fire.
\end{abstract}

\section{INTRODUCTION}

Preparedness planning has traditionally been modeled through tactical analysis focused on resource protection [1]. We offer an approach that significantly deviates from these methods and focusses on strategic analysis at the landscape level by integrating loss mitigation with the management of beneficial wildland fire (BWF). This more comprehensive and broad scale analysis strengthens the ability to support strategic budgeting and planning. A key rationale behind integrating these preparedness program components is that the same fire management resources can be used for both (with minor differences); they use similar crews, equipment and planning. This implies that the two components are directly tied through the production process. Even if they are budgeted separately, their budgets are unavoidably interrelated.

This more inclusive approach reflects current interagency policies that recognize the importance of incorporating the beneficial impacts of fire in a performance-based system $[2,3]$ using a common performance metric. For a given preparedness budget (B) the beneficial impact of fire can be added to the loss mitigation as the expected values for both loss mitigation and ecosystem improvement:

$$
\mathrm{V}=\mathrm{V}^{\mathrm{IA}}(\mathrm{B})+\mathrm{V}^{\mathrm{BWF}}(\mathrm{B})
$$

Where $\mathrm{V}$ denotes the expected value added from mitigating potential fire losses through initial attack $\mathrm{V}^{\mathrm{IA}}(\mathrm{B})$ and from BWF $\mathrm{V}^{\mathrm{BWF}}$. The term (B) denotes the amount of the preparedness 
budget. Methods for modelling the budget effect on loss mitigation through initial attack (IA) are described first followed by methods for modelling the beneficial effects of managing BWF.

\section{METHODS}

The methods apply the core concept that distance is fundamental to the performance and cost of IA and BWF components of the preparedness program when other landscape situations are similar. The methods are based on the consistent philosophy that distance of the cell to the decision point is central and the impact of the distance on performance can be managed through application of the budget with diminishing effect. For loss mitigation, we focus on the distance in time that a cell is to the dispatch location. For BWF, we focus on the distance that a cell receiving an ecosystem benefit is to its nearest downwind cell containing a highly valued resource to protect.

Both approaches are strategic in that no specific fire management resources or their activities are modelled. Both approaches are novel in that strategic level budget analysis has never been performed before, which abstracts away from individual resources and the tactics of fire management.

\subsection{Study site - sequoia and kings canyon national parks}

The methods are applied to data obtained from Sequoia and Kings Canyon National Parks (SKNP). The parks are located in the western Sierra Nevada mountain range in California. They contain about 360 thousand hectares of land comprising sequoia groves, montane forest, subalpine woodlands, oak woodlands and chaparral. SKNP maintains an active preparedness program providing ample data for the analysis.

For application of the preparedness analysis, we first need to identify where value is lost by wildland fire and where it can be gained. This assessment was performed on the SKNP landscape using the MARS valuation system [4] combined with the burn probability and fire behaviour information generated in the STARFire application [5]. STARFire's output provides the locations of cells that would produce ecosystem benefit from burning ('green' cells) and those that would produce a loss ('red' cells) [6]. These locations are depicted in panels (a) and (b) in Fig. 1.

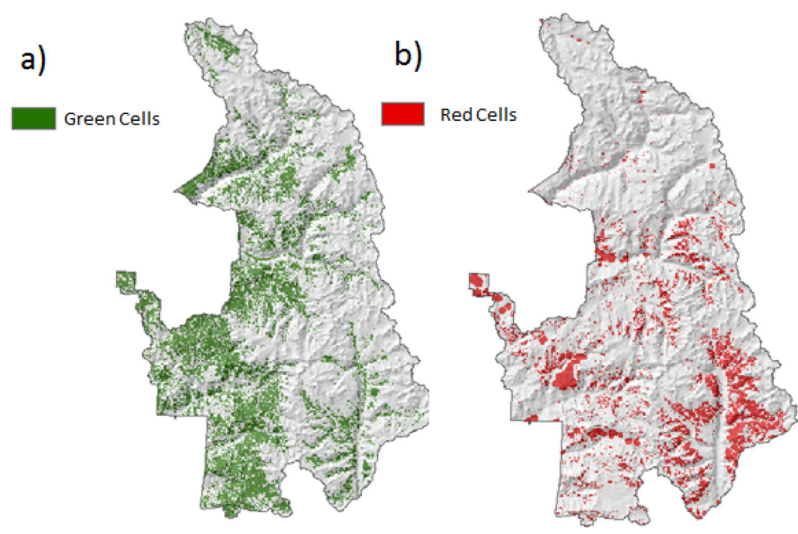

Figure 1: Location of cells that provide ecosystem benefit from burning ('green' cells) at SKNP (a) and cells that produce a negative outcome ('red' cells) (b). 


\subsection{Loss mitigation}

We define preparedness as being prepared for the fire season through planning, and availability of crews and equipment for initial attack in ways that reduce the probability that fires would continue beyond IA. Hence, we measure performance by the probability of fires reaching beyond the customary 300 acre IA standard. A different size standard is readily substituted for modelling purposes or to reflect standards consistent with other agencies or governments.

We assume that the probability of containing fires within the IA standard depends upon:

1. how close in distance in time the cell is to its nearest dispatch location, the rate of spread (ROS), at the ignition cell,

2. the amount of time required for a fire to consume 300 acres or more (given itsROS), the effort or intensity of preparedness resources applied as reflected by the preparedness budget,

3. a cell's currently measured ignition probability,

4. the calculated probability of fire reaching beyond IA (escape probability),

5. and the percentage rate that increases in the preparedness budget to reduce the probability of success of an IA. This is measured from an external IA simulator currently used by the US Bureau of Land Management.

The ignition probability represents an upper limit on the probability of a fire reaching past initial attack. For example, at a zero preparedness budget, the ignition probability equals the escape probability suggesting that the fire would not be fought or it would be fought by other means such as extended attack resources. The escape time defines how much time is available to fight the fire before it exceeds the IA standard of 300 acres, or a standard set by the land management agency. If the standard is reached the assumption is that preparedness resources were unable to arrive before the fire became an extended attack event. Conversely, if resources arrive quickly, then the probability of the fire reaching beyond IA is reduced depending upon how quickly resources arrive and upon the intensity of effort applied through the preparedness budget.

This requires calculations for estimating the amount of time a fire can burn before it reaches escape size. The term $\mathrm{T}_{\mathrm{c}}$ reflects the time required for a fire to spread across the long radius a $300 \mathrm{~m} \mathrm{2:1} \mathrm{ellipse} \mathrm{[7]} \mathrm{as} \mathrm{expressed} \mathrm{in} \mathrm{(2).}$

$$
\mathrm{T}_{\mathrm{c}}=\frac{2 * \sqrt{\frac{\mathrm{SQm}}{2 \pi}}}{\operatorname{ROS}_{\mathrm{c}}}
$$

In (2), SQm denotes the fire size limit for initial attack success in square meters and ROS $_{c}$ denotes the ROS in meters per minute for the particular cell (c). A value for $T_{c}$ is calculated for each cell to reflect variation in spread rates by cell.

To calculate resource arrival time for each cell to its nearest dispatch location, several spatial layers provided by the National Park Service (NPS) were used. A cost layer that identified the elapsed time of travelling to any cell on the landscape considering terrain, topography, roads and other factor was coupled with ground dispatch locations to calculate the least accumulative cost distance for each cell on the landscape to the nearest ground dispatch location 
using ESRI's [8] 'Cost Distance' tool. The resulting output identifies the time (in hours) to get from any location on the landscape to the closest ground dispatch location.

Next, the preparedness budget is introduced to reflect the intensity with which IA resources are applied to the cell under the assumption that increased budgets promote more intensity of dispatch to any given cell. For example, at a zero budget, there is no dispatch and there is no reduction to the ignition probability that is used as a proxy for the probability that the fire will escape IA $\left(\mathrm{P}^{\mathrm{IA}}\right)$. As the budget is increased, the calculated value for $\mathrm{P}^{\mathrm{IA}}$ declines. As the budget increases to a very large number, $\mathrm{P}$ IA approaches zero. To implement these concepts, we assume that each additional $\$ 100,000$ increase in the budget reduces $\mathrm{P}^{\mathrm{IA}}$ by five percentage points across the IA portion of the preparedness program. By knowing the percentage rates of decline in the IA success rate, and the initial ignition probability, we can arrive at a solution for the effect of budget increases on initial attack success rate.

The value of the loss mitigation component of preparedness can be expressed by the reduction in the expected loss for a particular cell (n) of a set N. N represents all cells that would produce a loss if burned. Equation 3 sums the expected value added across the landscape:

$$
\mathrm{V}^{\mathrm{IA}}(\mathrm{B})=\mathrm{V}^{\mathrm{IA}}(0)-\sum_{\mathrm{n}=1}^{\mathrm{N}} \mathrm{P}_{\mathrm{n}}^{\mathrm{IA}}(\mathrm{B}) * \mathrm{~V}_{n}
$$

\subsection{Managing beneficial wild fire (BWF)}

We define the management of beneficial wild fire (BWF) as the management of wildfire for resource or ecological improvement. Budgeting for BWF management is for the purpose of enabling the allocation of fire resources to manage fires for these purposes. Higher preparedness budgets enable expansion of the BWF program at a given planning unit. We model the size of the program as the number of cells that can be managed for BWF annually.

To develop the BWF portion of the value added function, we make a set of parallel (to the loss mitigation section) assumptions regarding the impact of the budget.

1. We assume that beneficial effects can be managed through the preparedness budget and that expending the budget produces increased benefits at a diminishing rate.

2. We assert that the cost of managing a cell for benefit is related to the cell's proximity to a cell with detrimental implications. Specifically, the closer the candidate BWF cell is to an undesirable cell (in a downwind direction), the more it will cost to manage for BWF purposes.

The program benefit is defined as the cellular burn probability (BP) times the positive value produced by burning the green cell $(\mathrm{V}+)$. This is modelled for each cell across the program membership (M) (as defined below) and summed across the landscape. The calculation of BWF benefit is specified as in (4) where the value of the budget for beneficial BWF purposes $\mathrm{V}^{\mathrm{BWF}}(\mathrm{B})$ is defined as:

$$
\mathrm{V}^{\mathrm{BWF}}(\mathrm{B})=\sum_{\mathrm{m}=1}^{\mathrm{M}} \mathrm{PB}_{\mathrm{m}} * \mathrm{~V}_{\mathrm{m}}^{+}
$$

To estimate the benefit of a BWF program as in (4) we need to know which cells and how many of them are included in the program across the landscape. Program membership is an abstraction used to model the nature and size of the BWF program. A planning unit with a small budget will have little opportunity to use BWF especially as fires are closer to values to 


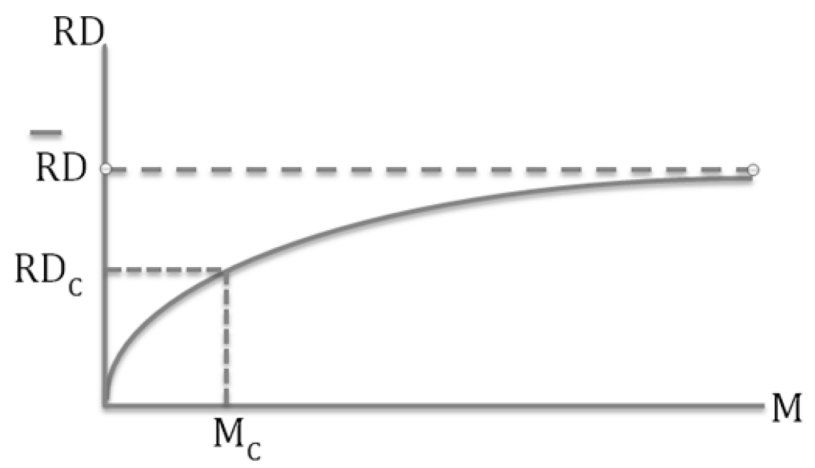

Figure 2: Increasing budgets result in more green cells participating in BWF membership (M) because we can reduce the distance (RD) to loss-producing cells. RD bar denotes full reduction in distance where all cells $(\mathrm{M})$ are included in the program membership.

protect. An extremely poor unit would only enable BFW in very remote areas. As the budget grows, it will be able to increase membership in the BFW program to include green cells closer to values to protect. This abstraction enables a pragmatic modelling of the BWF benefit as a function of size of the preparedness budget. We first assume that membership diminishes with budget increases (diminishing returns) using a constant percentage rate. The maximum potential membership is defined by the total number of beneficial (green) cells on the landscape (Fig. 1). The implicit assumption for the measurement is that with an infinite budget we could manage all green cells as part of the BWF program (Fig. 2).

The cost of the program is determined by the preparedness budget. As the budget increases, the size of the program is expanded by increasing the number of cells that qualify for membership. Conversely, as the budget decreases, so does membership. To define the relationship between membership and budget, the entire landscape is evaluated to calculate the maximum number of green cells that could be included in the BWF management program under an infinite budget. As the budget increases, more cells that are closer to a red (loss-producing) cell are included in the analysis. The program is built by adding the least expensive cells first and progressing to more expensive cells as the budget expands. At very high budgets more crews, equipment, and support are available to protect highly valued assets, enabling more cells to be included in the program. For example, cells that are very distant from a resource at risk are included in the BWF program membership at low budgets because they are inexpensive to manage relative to cells that are close to values at risk. At high preparedness budgets, the cell membership increases by reducing the distance (RD) that BWF member cells can be to loss-inducing cells. The population of cells included in the BWF program (M) depends upon the distance (D) from any cell to its nearest downwind red cell. This distance is defined as a function of the preparedness budget (B) such that: $M=M(D(B))$.

Next, the percentage rate of membership increase with respect to budget increases is estimated. Using historical fire perimeters, the number of green cells that have burned annually under approximately the current annual budget is determined. The number of green cells within a fire perimeter greater than 300 acres are tallied for each fire perimeter (Fig. 3 - panel (a)) and annualized by the number of fire years. The green cell annual budget is used in the calculation. 

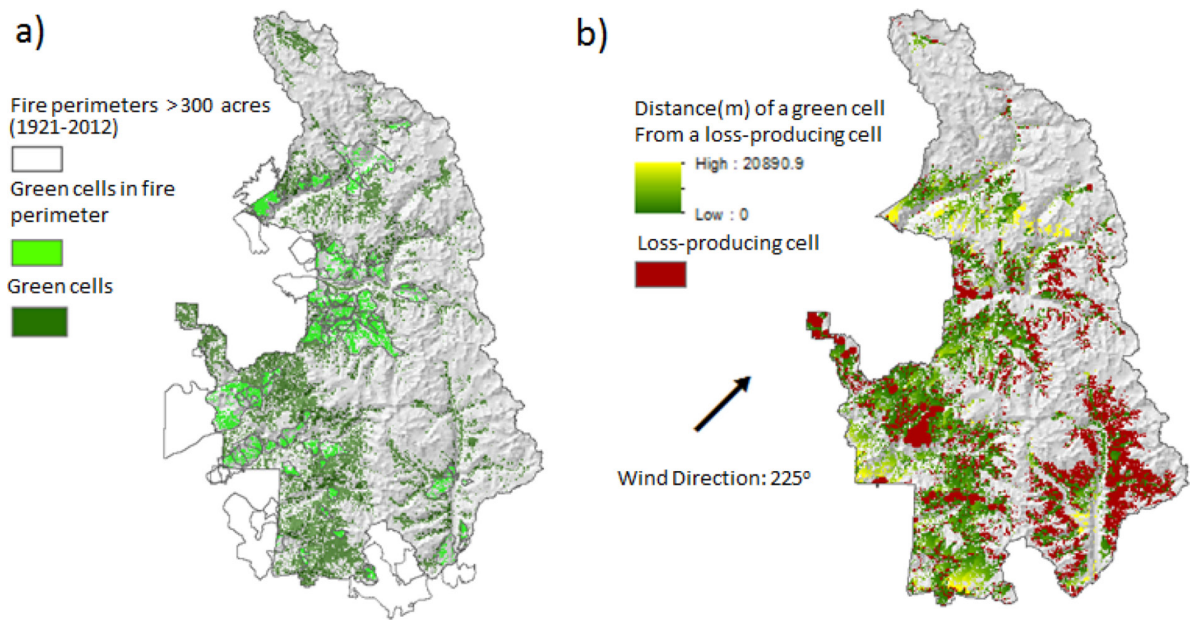

Figure 3: In panel (a) the green cells that have historically burned are represented in light green. In panel (b) green cells closest to a loss-producing cell are represented in dark green. As the distance increases the colour decreases to light yellow.

Next, the membership of the BWF program was estimated and established as a function of distance in time to the nearest downwind cell. Wind directions were estimated for the SKNP landscape in WindNinja [9]. WindNinja is a public domain software developed by the USDA and Forest Service for wildland fire applications to estimate spatially varying wind fields using an average surface wind direction and speed. The downwind distance from a lossproducing cell was calculated using ESRI's [8] Path Distance tool. The WindNinja wind layer was used as the horizontal factor and the average wind direction value as the cut angle setting. The resulting output (Fig. 3 - panel (b)) contains the downwind distance (meters) of any green cell from its nearest loss-producing red cell.

2.4 Using return on investment to measure the performance of each preparedness component

We assessed the return on investment (ROI) from preparedness by incorporating eqns (3) and (4) into the STARFire spatial fire planning and budgeting system [5]. STARFire provides the ability to estimate the ROI from fire management actions [10]. By incorporating the value added from the loss mitigation in eqn 3 and the value added from BWF in eqn 4, we can estimate how much value added each preparedness component contributes to SKNP's landscape by budget level (cost).

\section{RESULTS}

The dual purpose of a preparedness budget were quantified at SKNP across a wide range of budget levels as shown in Fig. 4 on the x-axis. In Fig. 4, the y-axis measures the value added for each planning or budget alternative. The bottom section of each bar shows the value added by the loss mitigation portion of the preparedness program. The middle portion of the bars show the value added by the BWF portion of the preparedness program. The highest portion of the bars show how much potential value still remains to be gained after the preparedness 


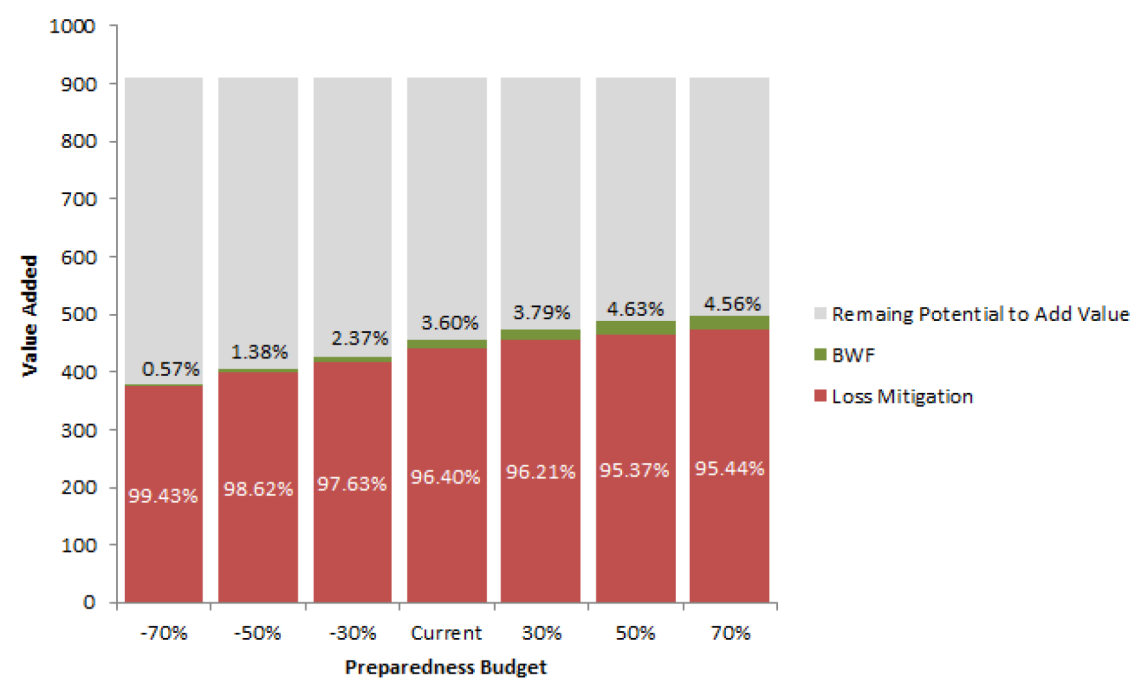

Figure 4: Value added to the landscape by the preparedness program funded at alternative levels.

program has been implemented. There is the potential to add value from other programs such as fuel treatments (not addressed in this paper). At this park, the loss mitigation portion of the preparedness program is much greater than the BWF portion. These results reflect the fact that SKNP is home to some of the nation's most famous natural resources as well as many highly valued developments that are critically important to protect from wildland fire.

\section{CONCLUSIONS}

The results enable wildland fire officials to address and quantify the value added and ROI of the integrated preparedness program. They can also analyse the IA and BWF components of their preparedness programs strategically, knowing how the two programs can be managed and appropriated in complementary ways. Previous approaches that only addressed initial attack modelling to reduce detrimental fire events have important strengths, but they lack the ability to integrate the impact of BWF as an important part of ecosystem management. This research shows for the first time, that a strategic level approach is potentially viable for IA and for integrating IA with BWF. Failure to integrate the two programs can understate the ROI to the preparedness program. This research shows that this understatement can be in the neighbourhood of $3.6 \%$ for landscapes similar to SKNP, but it could be considerably higher where managers rely more heavily on the management of BWF to accomplish ecosystem objectives. The results further suggest that with more comprehensive modelling, planning options can be better tailored to the planning unit to improve decision-making, budgeting, and the defensibility of budget expenditures and appropriation requests.

\section{REFERENCES}

[1] Wei, Y., Bevers, M. \& Belval E.J., Designing seasonal initial attack resource deployment and dispatch rules using a two-stage stochastic. Programming procedure. Forest Science, 61(6), pp. 1021-1032, 2015.

http://dx.doi.org/10.5849/forsci.14-182 
[2] United States Congress, FLAME act of 2009, H.R. 2996. In One Hundred Eleventh Congress of the United States of America, 2009.

[3] United States Congress, Healthy forests restoration act of 2003, H.R. In 1904, One Hundred Eight Congress of the United States of America, 2003.

[4] Rideout, D.B., Ziesler, P.S., Kling, R., Loomis, J.B. \& Botti, S.J., Estimating rates of substitution for protecting values at risk for initial attack planning and budgeting. Forest Policy and Economics, 10(2), pp. 205-219, 2008. http://dx.doi.org/10.1016/j.forpol.2007.10.003

[5] Manley, J., Rideout, D.B., Wei, Y. \& Botti, S.J., STARFire: decision support for strategic integration of wildland fules and unplanned ignitions. In Proceedings of the 3rd Fire Behavior and Fuels Conference, International Association of Wildland Fire, Spokane, WA, 2011.

[6] Rideout, D.B., Ziesler, P.S. \& Kernohan, N., Valuing fire planning alternatives in forest restoration: using derived demand to integrate economics with ecological restoration. Journal of Environmental Management, 141, pp. 190-200, 2014. http://dx.doi.org/10.1016/j.jenvman.2014.03.023

[7] Mees, R.M., Simulating initial attack with two fire containment models. USDA Forest Service Research Note PSW-378, 1985.

[8] ESRI, ArcGIS Desktop: Release 10.2.2, Redlands, CA: Environmental Systems Research Institute, 2016.

[9] WindNinja, 2016., Retrieved from http://www.firelab.org/document/windninja-software

[10] Rideout, D.B., Wei, Y. \& Kirsch, A., Optimal allocation of initial attack resources to multiple wildfire events. International Journal of Safety and Security Engineering, 1(3), pp. 312-325, 2011. http://dx.doi.org/10.2495/SAFE-V1-N3-312-325 